\title{
A New Definition for the Ca4227 Feature: Is Calcium Really Underabundant in Early-type Galaxies?
}

\author{
L. C. Prochaska \\ Department of Physics and Astronomy, CB 3255, University of North Carolina, Chapel Hill, NC \\ 27599 \\ Electronic mail: chaska@physics.unc.edu \\ James A. Rose \\ Department of Physics and Astronomy, CB 3255, University of North Carolina, Chapel Hill, NC \\ 27599 \\ Electronic mail: jim@physics.unc.edu \\ Ricardo P. Schiavon \\ Department of Astronomy, University of Virginia, P.O. Box 3818, Charlottesville, VA 22903-0818 \\ Electronic mail: ripisc@virginia.edu
}

\begin{abstract}
We have investigated the abundance of calcium in early-type galaxies by measuring the strength of the Ca I 4227 absorption line in their integrated spectra. The database used is the large sample of early-type galaxy integrated spectra in Caldwell, Rose, \& Concannon (2003). We have measured Ca abundances from the Ca I 4227 feature both by using the Lick Ca4227 index and also by defining a new index, Ca4227 ${ }_{r}$, that avoids the CN4216 molecular band in the continuum on the blueward side of the line. With the new index definition we measure Ca abundances that are systematically $\sim 0.3$ dex higher than with the Lick Ca4227 index. The result is that with the new index definition we obtain higher $[\mathrm{Ca} / \mathrm{Fe}]$ abundances in early-type galaxies which are more consistent with their well known $[\mathrm{Mg} / \mathrm{Fe}]$ over-abundances. Hence, we suggest that Ca might be slightly enhanced, relative to Fe, in early-type galaxies.
\end{abstract}

Subject headings: galaxies: abundances — galaxies: evolution — galaxies: stellar content

\section{Introduction}

Accurate determinations of the chemical abundances in galaxies, based on observations of their integrated spectra and on population synthesis models, provide a fundamental contribution 
to deciphering the evolution of galaxies. While initial work focused primarily on determining an overall heavy element abundance in galaxies, for some time now it has been recognized that the chemical abundance patterns of other galaxies can be distinctly non-solar. The most striking example of non-solar abundance ratios is evident in the enhanced $[\mathrm{Mg} / \mathrm{Fe}] \sim+0.3$ observed in the more massive early-type galaxies (Peletier 1989, Worthey et al. 1992, Davies et al. 1993, Kuntschner 2000, and references therein). It is generally concluded that the enhanced magnesium-to-iron abundance implies an overall enhancement of $\alpha$ elements relative to iron. Enhanced $[\alpha / \mathrm{Fe}]$ could result from a rapid star formation and chemical enrichment history in massive early-type galaxies, when compared to the $\sim 5$ Gyr enrichment history of the pre-solar nebula (Worthey et al. 1992, Matteucci 1994, Thomas et al. 1999, Kuntschner 2000, and references therein). A counterpart to the $\alpha$-element enhancement in galaxies is found in Galactic globular clusters, for which similar non-solar $[\alpha / \mathrm{Fe}]$ is seen, and for which rapid enrichment histories are expected, given their very old ages.

Given the scenarios discussed above, it has come as a surprise that several recent studies of the calcium abundances in early-type galaxies indicate that calcium does not track its $\alpha$-element companion $\mathrm{Mg}$, but rather that $[\mathrm{Ca} / \mathrm{Mg}] \sim-0.3$, and $[\mathrm{Ca} / \mathrm{Fe}] \sim 0.0$ (Vazdekis et al. 1997, Worthey 1998, Trager et al. 1998, Henry \& Worthey 1999, Thomas et al. 2003, and references therein). In addition, Ca line strengths show a weak correlation with galaxy velocity dispersion (thus galaxy mass), while $\mathrm{Mg}$, as measured by the Lick Mg $b$ index, exhibits a steep trend with $\sigma$. In short, unlike other $\alpha$ elements, calcium abundances seem to track those of iron in giant early-type galaxies. Thomas et al. (2003) have investigated several explanations for how Ca can be decoupled from other $\alpha$ elements. Except for the scenario in which $\mathrm{Ca}$ is preferentially depleted onto dust grains, these scenarios all require rather extreme conditions in terms of the initial mass function and/or star formation rates, thus implying important modifications to previously assumed star formation histories in galaxies.

The situation concerning calcium in galaxies is rendered even more puzzling by the fact that little evidence exists for an underabundance of calcium with respect to other $\alpha$ elements in our Galaxy, even among the moderately metal-poor field stars in the solar neighborhood and thick disk stars which exhibit strong non-solar abundance ratio trends (Wheeler et al. 1989; Edvarsson et al. 1993; Reddy et al. 2003). Some evidence has been reported for calcium (and titanium) underabundances relative to the other $\alpha$ elements in Galactic bulge giants (McWilliam \& Rich 1994). Furthermore, Zoccali et al. (2004) report low $[\mathrm{Ca} / \alpha]$ in giants in the metal-rich globular cluster NGC 6528, but both Carreta et al. (2001) and Origlia, Valenti, \& Rich (2005) find that Ca tracks the other $\alpha$ elements in this cluster. Discrepancies among these studies undoubtedly result from the difficulty in conducting reliable high dispersion abundance analyses for relatively cool, low-gravity stars in regions of high and variable reddening.

Clearly, the abundance of calcium potentially provides a crucial insight into their chemical enrichment histories. The low calcium abundances estimated in galaxies come from measurements of three different spectral features. The first is the Ca I 4227 resonance line, which has been 
measured in the Lick spectral index system, as defined in Worthey et al. (1994), and then redefined in Trager et al. (1998). A second feature is the Ca 4455 line, which has been measured in integrated spectra of early-type galaxies by, e.g., Trager et al. (1998) and Vazdekis et al. (1997). Third, there is the Ca triplet at $\approx 8500 \AA$, which has been extensively studied in stars, globular clusters, and early-type galaxies (Cohen 1979, Faber and French 1980, Alloin and Bica 1989, Idiart et al. 1997, Schiavon, Barbuy, \& Bruzual (2000), Cenarro et al. 2001, Vazdekis et al. 2003). Each of these spectral features has associated difficulties in regard to obtaining a clean abundance measurement of calcium. The Ca4455 feature is a blend with Fe (Tripicco and Bell 1995), which, as noted above, is known to be underabundant relative to $\alpha$ elements in early-type galaxies. The Ca triplet in the red has three associated problems. The first is that while measurements of the triplet correlate well with metallicity in metal-poor systems, little abundance sensitivity is seen at higher metallicities (Alloin and Bica 1989, Vazdekis et al. 2003, and references therein). Second, because the feature is located far in the red, the stars dominating the integrated light of galaxies at the triplet come from the upper part of the red giant branch (RGB) and the asymptotic giant branch (AGB). Due to the uncertainties in the modelling of the atmospheres of such cool giant stars, their elemental abundances are highly uncertain. In fact, even the iron abundances of the $\mathrm{M}$ giant stars included in the stellar libraries underlying current stellar population synthesis models are poorly known, if not entirely unknown. In addition, the mean temperature of the stars dominating the light at $8500 \AA$ will be highly dependent on accurately modeling the luminosity function along the upper RGB and AGB, which is difficult to achieve given the vagaries of mass loss and sporadic episodes of elevated nuclear burning. Third, the Ca triplet is potentially contaminated by $\mathrm{TiO}$ features and by the Paschen lines (Alloin and Bica 1989, Cenarro et al. 2001), which has led to the definition of a $\mathrm{CaT}^{*}$ index that is meant to minimize those problems (Cenarro et al. 2001). Finally, the Ca4227 line lies in a crowded spectral region in the blue, making it difficult to define an index that cleanly measures the abundance of $\mathrm{Ca}$. The situation is exacerbated by the presence of the strong CN4216 band just blueward of the Ca4227 feature. To further underscore the ambiguities surrounding what the Ca II triplet and the CaI4227 features actually measure, Cenarro et al. (2004) have reported divergent behavior in $\mathrm{Ca}$ abundances extracted from the two features in field versus Coma cluster galaxies.

In this paper we focus on the Ca4227 feature in the integrated spectra of early-type galaxies. In $\S 2$ we discuss the sample of galaxy spectra and population modeling procedures that form the basis of our study. In $\S 3$ we discuss the difficulties in measuring the Ca4227 line and define a new index that is designed to minimize the problems. In $\S 4$ we present the results on Ca abundances in galaxies obtained with our new index, and show how these results differ substantially from those obtained with the previous Ca4227 index. In $\S 5$ we present systematic tests of our new index. In $\S 6$ the implications of our results are summarized. 


\section{Observational Data and Models}

\subsection{Observations}

The observational data used in this paper consist of spectra of 175 early-type galaxies obtained with the $1.5 \mathrm{~m}$ Tillinghast Telescope, FAST spectrograph, and a Loral 512 x 2688 pixel CCD (Fabricant et al. 1998) at the F. L. Whipple Observatory. The spectra were obtained at a dispersion of $0.75 \AA /$ pixel and a resolution of $3.1 \AA$ FWHM, and are further described in Caldwell, Rose, \& Concannon 2003 (hereafter CRC). A key point is that the galaxy sample covers early-type galaxies over an extensive range in velocity dispersion, $\sigma$, from $50 \mathrm{~km} \mathrm{~s}^{-1}$ to more than $300 \mathrm{~km} \mathrm{~s}^{-1}$. As in CRC we restrict ourselves to the galaxies in the sample with $\sigma \leq 230 \mathrm{~km} \mathrm{~s}^{-1}$.

\subsection{Population Synthesis Models}

To extract useful information about the Ca abundances in galaxies, as well as abundances of other $\alpha$-elements and of $\mathrm{Fe}$, requires comparison of the observed line strengths in galaxy spectra to the predictions of a grid of stellar population synthesis models covering a range in age and chemical composition. In this paper we use the population synthesis models of Schiavon (2005) which are fully described in that paper. Briefly, the population models rely on the Padova isochrones of Girardi et al. (2000) and Salasnich et al. (2000) and a new set of fitting functions to the spectral indices used in this paper. Equivalent widths of spectral indices are measured from the spectral library of Jones (1999). Theoretical stellar parameters are combined with these equivalent widths and polynomial fitting functions are computed. Predictions of integrated line indices are then generated by combining these fitting functions with the Padova theoretical isochrones. Further details can be found in Schiavon (2005).

\section{A New Ca4227 Index}

\subsection{Problems in Defining a Ca4227 Index}

As mentioned in the Introduction, a critical issue for reliably measuring element abundance ratios in the integrated spectra of galaxies is to isolate the absorption feature of interest from other

spectral features that may be overlapping in wavelength. As well, since an accurate assessment of the line strength requires the location of the stellar continuum on either side of the absorption line, excessive contamination of the nearby continuum is in principle to be avoided. The Ca4227 feature is in a particularly problematic wavelength region from two considerations. First, line crowding is especially severe in the blue, hence it is difficult to isolate the Ca4227 line core and its potentially extensive wings from other features in that region. The result is that a truly clean definition of Ca4227 is quite difficult to achieve. The problem of line crowding is illustrated in Fig. 1, where 
we have plotted the spectra of three representative stars. The spectra have been taken from the "Indo-US Library of Coude Feed Stellar Spectra" (Valdes et al. 2004), which consists of spectra of 1273 stars at a resolution of $\sim 1.2 \AA$ FWHM. The spectrum on the bottom is that of an M dwarf, and illustrates the extensive wings of Ca4227 in cool dwarfs. Thus to obtain an assessment of the complete equivalent width of the line requires moving the neighboring continuum bandpasses out by at least $30 \AA$ from the line center. The middle and top spectra are respectively from a G5V and a KOIII star, which are the spectral types characteristic of the stars that dominate the integrated light of stellar populations in the blue spectral region. They indicate the degree of line crowding characteristic of stars that contribute to the integrated light of intermediate-age and old stellar population in the blue spectra region. Moreover, they illustrate a fundamental issue in defining the Ca4227 index. Namely, at only $10 \AA$ blueward of the Ca4227 line center lies a strong feature which is due to a CN bandhead at $\lambda \sim 4216 \AA$ Tंhe CN4216 molecular band consists of an extensive series of features that are most pronounced at the bandhead, but extend blueward for $\sim 100 \AA$. The CN4216 band, which is prominent in cool low-gravity stars, is demarcated by the wedge at the top of Fig. 1. It is clearly not feasible to find anything like a true continuum around the Ca4227 line.

In principle, of course, it should not really matter whether a true continuum is established for defining a spectral index, as long as the observed index in a galaxy integrated spectrum is compared to reliable stellar population models, based on a stellar library that accurately reflects the spectra of the constituent stars in the galaxy. In practice, however, the stellar abundance patterns in earlytype galaxies (at least in the more massive ones), appear to be distinct from the abundance pattern in Solar Neighborhood stars, being enhanced in the $\alpha$ elements such as Mg (Worthey et al. 1992, Davies et al. 1993, Kuntschner 2000, and references therein). As shown by Trager et al. (1998), the strengths of $\mathrm{CN}$ bands in $\alpha$-enhanced, massive early-type galaxies tend to be stronger than predicted by the models, probably due to an overabundance ofnitrogen (Worthey 1998). Therefore, the strength of the CN4216 band may be underestimated in population synthesis models for the integrated spectrum of an early-type galaxy, due to the use of a spectral library based on Solar Neighborhood stars. The result will be an overestimate of the predicted strength of Ca4227 at a given "metallicity", due to an underestimate of the contamination from $\mathrm{CN}$ in the continuum

bandpass to the blue of Ca4227. Consequently, the derived Ca abundance in the galaxy will be underestimated.

\subsection{Definition of the $\mathrm{Ca}^{2} 227_{r}$ Index}

From the discussion above, it should be evident that two issues need special consideration in defining an index that best isolates the Ca4227 absorption line. The first is that the generally high degree of line crowding in the region of Ca4227 makes it advantageous to use the narrowest possible bandpass in isolating Ca4227 from other contaminating features. On the other hand, we must take into account that the line wings can be quite extensive, as illustrated by the $\mathrm{M}$ dwarf in Fig. 1. However, from the standpoint of galaxy (and globular cluster) integrated spectra, M dwarfs 
contribute little to the integrated light, hence the necessity for measuring the wings of Ca4227 is greatly overstated from the $\mathrm{M}$ dwarf spectrum. Regardless, there is also the danger in too narrowly confining the Ca4227 measurement to only the central few $\AA$ such that the results will then be highly sensitive to velocity broadening effects in the galaxy spectrum, which redistribute flux from the line core into the wings. Moreover, very narrow index passbands require very high $\mathrm{S} / \mathrm{N}$ spectra in order to be measured accurately. While in principle it is possible to accurately measure the velocity dispersion of the galaxy, as well as the intrinsic resolution of the spectrum, an index highly dependent on resolution effects is best avoided. As a compromise, we have chosen to measure a bandpass centered on Ca4227 that is illustrated by the dotted line in Fig. 2. There we have replotted the spectrum of the same K0III star from Fig. 1, and also, for reference, plot the central bandpass of the Lick Ca4227 index as a solid line. Note that our bandpass is similar to the Lick bandpass, with a slight shift in central wavelength.

The second consideration regards the placement of the pseudo-continuum bandpasses. The Lick Ca4227 blue and red continuum bandpasses are represented by solid lines at the top of Fig. 2 . Note that although the blue continuum bandpass is quite narrowly defined, and situated close to the Ca4227 feature, it unavoidably falls into the CN4216 molecular band. The only way to avoid the CN band would be to define a very narrow blue continuum bandpass that isolates the peak just longward of the CN bandhead at $4216 \AA$. This method was followed in Rose (1994). However, it is subject to the above-mentioned resolution effects, in that increased velocity broadening will remove flux from the localized pseudocontinuum peak. Consequently, the approach we have chosen for the new $\mathrm{Ca}_{2227}$ ( $r$ for red bandpass) index is to give up the idea of a blue continuum bandpass altogether. Instead, we have defined a red continuum bandpass, as denoted by the dotted line redward of Ca4227, and assume a flat continuum in the region of Ca4227 in calculating the pseudo-equivalent width. In effect, the Ca4227 $r$ index is simply the mean flux ratio between line and continuum bandpasses, but conveniently recast in terms of an equivalent width. The one disadvantage of this method is an increased sensitivity to poor flux calibration of the spectra and/or reddening (see $\S 5$ ). The exact wavelength limits of the Ca4227 $r$ and the Lick Ca4227 indices are given in Table 1.

\subsection{Measurement of Spectral Indices}

In this paper we make use of a number of key spectral line indices that are defined in the "Lick" system by Worthey et al. (1994). In particular, we use the Lick $\mathrm{H} \beta, \mathrm{Mg}$ b, Fe4383, and Ca4227 indices, in addition to our newly defined Ca4227 $r$ index. For the Lick indices we use the bandpass definitions given in Worthey et al. (1994). In the true Lick system, as it originated with the IDS detector, the spectral resolution varies with wavelength (as specified in Worthey \& Ottaviani 1997), and the spectra are on the instrumental response of the IDS. On the other hand, the CRC03 galaxy spectra used in this study are flux-calibrated, and are taken at a spectral resolution of 3.1 $\AA$ FWHM and then all spectra are smoothed to a common velocity broadening, $\sigma$, of $230 \mathrm{~km} \mathrm{~s}^{-1}$ (see CRC03 for details). It turns out that this effective resolution of the galaxy spectra, at both 
$\mathrm{H} \beta$ and $\mathrm{Mg} b$, almost precisely matches the resolution of the Lick system at those indices. To place ourselves closer to the Lick system, before calculating the Fe4383 and Ca4227 indices we smoothed the galaxy spectra by an extra amount to attain the same resolution as the Lick system. Note, however, that our results are not exactly on the true Lick system, since the galaxy spectra are flux-calibrated, while the Lick system, as mentioned above, is based on the IDS instrumental response. Thus although we refer hereafter to the "Lick" Ca4227, $\mathrm{H} \beta, \mathrm{Mg}$ b, and Fe4383 indices throughout the paper, the reader should note that we are not quite on the standard system. Due to the fact that the Lick indices always involve continuum bandpasses on both sides of the absorption feature of interest, the nature of the instrumental response has a very weak effect on the measured index. Note also that the models from Schiavon (2005) are also based on fitting functions from a flux-calibrated stellar spectral library. Thus although the library spectra have been smoothed by the appropriate amount for each Lick index, as in the case of our galaxy spectra, they are based on flux-calibrated stellar data. For the newly defined Ca4227 $r$ index, no additional smoothing has been carried out beyond getting all galaxy spectra onto a common velocity broadening of 230 $\mathrm{km} \mathrm{s}^{-1}$, in addition to the intrinsic resolution of the spectra of $3.1 \AA$ FWHM. We note that the model predictions by Schiavon (2005) for the Ca4227 $r$ index were computed for the exact same broadening as the data discussed here.

Finally, all of the indices, with the exception of $\mathrm{H} \beta$, are measured in the galaxy spectra using a modified version of the automated LECTOR program made publicly available by Alexander Vazdekis ${ }^{1}$. The $\mathrm{H} \beta$ indices are from CRC data which have been corrected for both the effects of emission and non solar abundance ratios. These correction procedures are documented in CRC. The measured Ca4227 $r$ and Lick Ca4227 index values for the spectral library of Jones (1999) used in the models are given in Table 2. The measured Ca4227 $r$ and Lick Ca4227 index values for each galaxy in the CRC sample along with the $\pm 1 \sigma$ uncertainties in the indices are given in Table 3 . Uncertainties in the Ca4227 $r$ indices were determined from the r.m.s. scatter in multiple exposures for many galaxies (see CRC for details). Uncertainties in the Lick spectral indices used by us are in principle already determined by CRC. However, since we have recalculated the Fe4383 and Lick Ca4227 indices after smoothing to the Lick resolution specified in Worthey \& Ottaviani (1997), we determined new $1 \sigma$ uncertainties by calculating the r.m.s. scatters in the Lick indices in multiple exposures for a subsample of the CRC galaxies. From this subsample we determined an overall correction factor from the published CRC uncertainties to those in our index determinations carried out at the Lick resolution. In the case of 47 Tuc, where multiple exposures were not available, we calculated the uncertainties from the signal to noise ratio per pixel integrated over the specified bandpass. The characteristic $\pm 1 \sigma$ error bars shown in Fig. 3 and all subsequent figures represent the mean errors for the entire CRC sample.

\footnotetext{
${ }^{1}$ http://www.iac.es/galeria/vazdekis/vazdekis_models.html
} 


\section{Results}

The primary goal of this study is to evaluate the behavior of $[\mathrm{Ca} / \mathrm{Fe}]$ and $[\mathrm{Ca} / \alpha]$ in galaxies. As was discussed in $\S 3$, there is reason to suspect that previous attempts to measure the calcium abundance through the Ca I $\lambda 4227$ feature may have been compromised by CN contamination in the blue continuum bandpass. Consequently, we have defined a new index, Ca4227 $r$, that is designed to remove the $\mathrm{CN}$ contamination problem. In this section we compare the behavior of the new calcium index with that of the Lick Ca4227 index.

\subsection{Empirical Trends}

We begin by examining the behavior of the old and new Ca indices in a strictly empirical sense, i.e., without reference to population synthesis modeling. Specifically, we use the large database of early-type galaxy spectra from CRC. One attribute of the CRC dataset is that it covers a large range in galaxy velocity dispersion (hence mass), thus we can examine trends in Ca versus other elements over a large mass range. Several major studies have established that the abundance of $\mathrm{Mg}$ increases substantially with galaxy velocity dispersion, $\sigma$, while the increase in Fe with $\sigma$ is less striking (Jorgensen 1997, Kuntschner 2000, and references therein). We reproduce the behavior of $\mathrm{Mg}$ and Fe with $\sigma$ found by $\mathrm{CRC}$ in their dataset in the bottom left and right panels of Fig. 3. We have used the $\mathrm{Mg} b$ and $\mathrm{Fe} 4383$ indices to track the abundances of $\mathrm{Mg}$ and Fe, respectively. There is a well-defined slope to the $\mathrm{Mg} b$ versus $\sigma$ relation that extends from the lowest to the highest- $\sigma$ galaxies. The only exceptions are those galaxies dominated by young stellar populations especially prominent at the low $\sigma$ end, which scatter to low $\mathrm{Mg} b$ values (to separate these young galaxies from the rest of the sample, we have denoted by open squares galaxies with $\mathrm{EW} \mathrm{H} \beta \gtrsim 3.0$ ). In contrast, the trend in Fe4383 versus $\sigma$ is not nearly so steep, and appears to flatten out at the high- $\sigma$ end. Again, there is a scatter of younger galaxies to low Fe4383, especially at the low $\sigma$ end. These trends are extensively discussed in CRC.

In the top left and right panels of Fig. 3 we illustrate the behavior of both the Lick Ca4227 index and our newly defined Ca4227 $r$ index. There is a noticeable difference in the trends with $\sigma$ for the two indices, in that the Lick Ca4227 index exhibits only a weak trend with $\sigma$, similar to that of the Fe4383 index, while Ca4227 $r$ exhibits a strong trend, similar to that seen in $\mathrm{Mg} b$. This striking difference in the empirical behavior of the two calcium indices suggests that indeed what one measures in the Ca4227 feature is critically dependent on the measurement strategy. However, to better assess how the actual extracted abundance of calcium depends on the index definition requires the use of population synthesis modeling, hence we turn to that approach in what follows. 


\subsection{Results from Population Synthesis}

The most widely used strategy for separating age from metallicity effects in the integrated spectra of galaxies is to plot a primarily age-sensitive Balmer line versus a metal-sensitive feature, and compare galaxy data to models of single stellar populations in these plots. Since the Lick $\mathrm{H} \beta$ index represents the most extensively used Balmer line strength indicator, we plot our calcium indices versus this spectral feature in Fig. 4. The left hand panel features the Lick Ca4227 index, while the right hand panel features our new Ca4227 $r$ index. For comparison, in the bottom left and right panels are plotted Fe4383 and $\mathrm{Mgb}$, respectively, versus $\mathrm{H} \beta$. In all panels the model population grids come from the Schiavon (2005) models described in $\S 2.2$, while the galaxy data are from the CRC spectra. The $\mathrm{H} \beta$ indices in the $\mathrm{CRC}$ data have been corrected both for the effects of emission and non solar abundance ratios. These correction procedures are documented in CRC. In the top left panel we basically reproduce the result of, e.g., Thomas et al. (2003), that the abundance of calcium appears to be rather low, especially in comparison with that of magnesium. While $[\mathrm{Mg} / \mathrm{H}]$ ranges from slightly sub-solar in low $\sigma$ galaxies to as much as +0.5 in the higher $\sigma$ galaxies, the Lick $\mathrm{Ca} 4227$ index indicates that $[\mathrm{Ca} / \mathrm{H}]$ is decidedly subsolar in most early-type galaxies. On the other hand, the Ca4227 $r$ plot indicates a substantially higher mean $[\mathrm{Ca} / \mathrm{H}]$ for early-type galaxies. Specifically, the mean $[\mathrm{Ca} / \mathrm{H}]$ is now somewhat higher than solar. Zeropoint uncertainties abound in population synthesis modeling, especially when comparing element abundances derived from one set of models with another one. Consequently, we consider the shift in the mean $[\mathrm{Ca} / \mathrm{H}]$ of $\sim+0.3$ from the Lick Ca4227 index to the Ca4227 $r$ index to provide the more compelling case for our assertion that the Lick $\mathrm{Ca}$ index definition may have led to an underestimate of $\mathrm{Ca}$ abundances in galaxies. However, there is also some evidence that the new Ca4427 $r$ zeropoint supplies a better representation of Ca abundances. Specifically, we have plotted as a large unfilled triangle the Ca4227 and Ca4227 $r$ indices for an integrated spectrum of the core of 47 Tuc, in which the spectrograph slit was trailed across the core diameter of the cluster. From high-dispersion spectroscopic abundance analyses of individual RGB stars, the $[\mathrm{Fe} / \mathrm{H}]$ value for 47 Tuc is determined to be $\sim-0.7$, while $[\mathrm{Ca} / \mathrm{Fe}$ ] estimates range from 0.0 to $\sim+0.2$ (Carretta et al. 2004). In the left hand panel the Ca abundance inferred for 47 Tuc is clearly far below the expected value of $[\mathrm{Ca} / \mathrm{H}] \sim-0.7$ (or perhaps -0.5 ), while in the right hand $\left(\mathrm{Ca} 4227_{r}\right)$ panel, the inferred $\mathrm{Ca}$ abundance is much closer to the expected value from the high dispersion spectroscopy of giants. Note that we expect a major effect on the Lick Ca4227-derived abundance, since roughly half the stars in the core of 47 Tuc are CN-strong (Norris \& Freeman 1979).

To further clarify the effect of $\mathrm{Ca}$ index definition on the derived $\mathrm{Ca}$ abundances, in Fig. 5 we plot the Lick Ca4227 index versus both Fe4383 (top left panel) and $\mathrm{Mg} b$ (bottom left panel) indices, while in the right hand panels we plot Fe4383 and $\mathrm{Mg} b$ versus the new $\mathrm{Ca} 4227_{r}$ index. The Schiavon (2005) model grid lines are compared to the CRC early-type galaxy data in the four panels. Since the models were computed under the assumption of solar abundance element ratios, not surprisingly, the model grid lines are highly degenerate in Fig. 5. In the Lick Ca4227 plots, the CRC galaxy data points lie on top of the grid lines in the Fe4383 diagram, whereas in the Mg $b$ plot 
the higher $\mathrm{Mg} b$ galaxies lie well separated from the grid. Hence these two panels reproduce the basic results that indicate an underabundance of Ca relative to other $\alpha$ elements (as represented here by Mg) (Vazdekis et al. 1997, Worthey 1998, Trager et al. 1998, Thomas et al. 2003). On the other hand, the opposite effect is seen in Ca4227 $7_{r}$, namely, that Ca is systematically slightly overabundant relative to Fe for the higher Ca4227 $r$ galaxies (top right panel) whereas it tracks $\mathrm{Mg} b$ very well (bottom right panel).

To summarize at this point, there is a systematic difference of $\sim 0.3$ between Ca abundances derived from the Lick 4227 index versus the new $4227_{r}$ index, in the sense that the Ca derived from the new index exceeds that from the old index. Population synthesis modeling also indicates that while the Ca abundance derived from the Lick index tracks that of Fe, the Ca4227 $7_{r}$-derived Ca abundances closely track that of $\mathrm{Mg}$, thereby indicating that with the new $\mathrm{Ca}$ index, Ca abundances appear to follow the trend exhibited by other $\alpha$ elements.

\section{Systematics}

In addition to assessing how sensitive a spectral index is to the abundance of the particular element being measured, it is important to determine how robust the index is to realistic errors of measurement arising from uncertainties in spectral resolution, wavelength zeropoint, and fluxcalibration.

\subsection{Instrumental Resolution}

We first consider to what extent realistic uncertainties in spectral resolution may compromise results on the abundance of Ca derived from the Ca4227 $r$ index. Two effects must be considered in this regard: uncertainties in determining the intrinsic resolution of the spectrograph/detector and uncertainties in determining the velocity dispersion of the particular galaxy.

Regarding the intrinsic spectral resolution, such information is typically generated from fitting Gaussian profiles to individual lines in an arc spectrum while focusing the spectrograph. While normally the focusing procedure is optimized to produce a uniform focus along the dispersion, it is not unusual to end up with up to a $5 \%$ variation in FWHM of the arc lines along the dispersion axis. Consequently, it is not necessarily valid to cite a single resolution for the entire spectrum. In addition, focus variations will occur during the night as a result of temperature changes and flexure of the spectrograph during a long exposure. Hence we consider a $5 \%$ error in the intrinsic resolution of the equipment to be a reasonable figure.

To simulate the effect of intrinsic resolution effects we utilized stellar spectra from the IndoUS Library of Coude Feed Stellar Spectra (Valdes et al. 2004) mentioned earlier in §3.1. Since this spectral library is sampled at much higher intrinsic spectral resolution (FWHM 1.2) than 
the galaxy spectra from CRC (FWHM 3.1), any resolution variations in the stellar library will be unimportant. Furthermore, the high signal-to-noise ratio ( $>100: 1$ per $0.4 \AA$ pixel) minimizes scatter in the results due to photon noise in the data. We Gaussian smoothed the stellar spectra to intrinsic resolutions of $2.95 \AA, 3.1 \AA$, and $3.25 \AA$ FWHM, and then further broadened them to a velocity dispersion of $230 \mathrm{~km} \mathrm{~s}^{-1}$. Thus the $3.1 \AA$ resolution spectra reproduce the resolution of our galaxy spectra (which are all smoothed as well to a common velocity dispersion of $230 \mathrm{~km} \mathrm{~s}^{-1}$ ) from which the Ca4227r index is measured, while the other two intrinsic resolutions simulate a $\pm 5 \%$ error in intrinsic resolution. In Fig. 6 we plot the resulting offset between the measured Ca4227 $r$ index of the spectra at a resolution of $3.1 \AA$ FWHM to spectra at 2.95 (left panel) and $3.25 \AA$ (right panel). This exercise has been carried out for stellar spectra covering a wide range in spectral type. As can be seen in Fig. 6, systematic shifts in the Ca4227 $r$ index are less than \pm 0.006 , which translates to a negligible $(\sim 0.01 \mathrm{dex})$ effect on the derived Ca abundance from the index.

\subsection{Velocity Broadening}

The observed spectral resolution is also degraded by velocity broadening of spectral lines in galaxies. Typical uncertainties in determining the velocity dispersion, $\sigma$, are $\pm 10 \mathrm{~km} \mathrm{~s}^{-1}$. To simulate that effect, we Gaussian broadened the same set of stellar spectra to velocity dispersions of $220 \mathrm{~km} \mathrm{~s}^{-1}, 230 \mathrm{~km} \mathrm{~s}^{-1}$, and $240 \mathrm{~km} \mathrm{~s}^{-1}$ (after first smoothing them to the intrinsic spectral resolution of $3.1 \AA$ FWHM of the CRC galaxy data). In Fig. 7 we show the systematic offset in the measured Ca4227 $r$ index that is caused by this amount of uncertainty in broadening. Here the effects are larger, but do not exceed \pm 0.03 . As can be seen from the average spacing of the model grid lines in Fig. 4, an uncertainty of \pm 0.03 in the Ca4227 $r$ index translates into an uncertainty of \pm 0.05 dex in the derived Ca abundance.

To obtain a more comprehensive idea regarding the sensitivity of Ca4227 $r$ to velocity dispersion, we Gaussian broadened a set of 5 stellar spectra (covering a range in spectral type) from $\sigma=0$ to $\sigma=300 \mathrm{~km} \mathrm{~s}^{-1}$. Ca4227 indices were measured for each and then normalized to the value of Ca4227r at $\sigma=0 \mathrm{~km} \mathrm{~s}^{-1}$. Such normalized indices are shown in Fig. 8 with each spectrum designated by a separate symbol.

\subsection{Wavelength Zero Point}

We have simulated the effect of uncertainty in the wavelength zero point of the spectra. Since the signal-to-noise ratio of galaxy spectra used for abundance determinations is generally high enough for a precise redshift determination, we expect that the primary source of wavelength zero point uncertainties will arise from spectrograph flexure. A half pixel uncertainty, which translates to $0.5 \AA$ at our spectral sampling, is likely a generous allowance for flexure errors. Accordingly, we 
have shifted the same sample of stellar spectra by $\pm 0.5 \AA$, and the results are plotted in Fig. 9. As in the case of velocity broadening, zero point shifts in wavelength can introduce an uncertainty of \pm 0.03 in the $\mathrm{Ca} 4227_{r}$ index, or \pm 0.05 dex in the derived Ca abundance. To further test the effect of wavelength zero point shift, we applied $\pm 0.5 \AA$ shifts to the CRC galaxy spectra. The results, while considerably noisier than for the very high $\mathrm{S} / \mathrm{N}$ ratio stellar spectra, are consistent with that derived for the stellar spectra.

\subsection{Flux Calibration and Reddening Effects}

Finally, given that the new Ca4227 $r$ index was defined using only one continuum bandpass, it is important to be aware of the sensitivity to uncertainties in the slope of the continuum. Uncertainties in slope could arise from flux-calibration errors or reddening effects. In the spirit of the above analysis, we simulated the effect by assuming an error in continuum slope that translates into an error in B-V color of $\pm 5 \%$. A slope error of this magnitude results in an offset in the measured $\mathrm{Ca} 4227_{r}$ index of \pm 0.01 , which reflects a change in derived $\mathrm{Ca}$ abundance of $\sim \pm 0.01$.

To summarize, realistic uncertainties in spectral resolution, wavelength zero point, and flux calibration lead to modest uncertainties in the Ca abundances derived from the Ca4227 $r$ index. The largest sources of uncertainty are wavelength zero point and velocity broadening which are at the level of \pm 0.05 dex in the derived Ca abundance. Flux calibration and instrumental resolution are less problematic.

\section{Summary}

Our analysis of the Ca I 4227 absorption line in the integrated spectra of galaxies has led to the following conclusions.

(i) We have defined a new index, Ca4227, that includes only a single continuum bandpass on the red side of the Ca feature at $4227 \AA$. We do not use a continuum bandpass blueward of the 4227 line due to the presence of the CN4216 molecular band in that region. The central bandpass

for our newly defined Ca4227r index is located between $4221 \AA$ and $4230.8 \AA$; the red continuum bandpass is between $4241 \AA$ and $4257 \AA$.

(ii) When applied to the integrated spectra of early-type galaxies, the new index produces a derived calcium abundance that is systematically greater than that determined from the Lick Ca4227 index of Worthey et al. (1994).

(iii) While previous investigations based on the Lick4227 index have found Ca in galaxies to be underabundant relative to other $\alpha$ elements, with the new index, Ca appears to present a behaviour that is more similar to that of other $\alpha$ elements, such as Mg. This result alleviates an otherwise difficult problem, either in nucleosynthesis or in preferential depletion onto dust (Thomas et al. 
2003), of lowering the Ca abundance with respect to $\mathrm{Mg}$.

(iv) The difference in $\mathrm{Ca}$ abundance from the two index definitions lies in a contribution to the blue continuum bandpass from the $\mathrm{CN}$ molecule, coupled with the non-solar abundance ratios in most early-type galaxies. Because Ca4227 $r$ uses only a red continuum bandpass, the CN contribution is avoided.

(v) We have investigated the effects of uncertainties in wavelength zeropoint, flux calibration, and spectral resolution. For reasonable uncertainties in each, we find that the effect on the measured Ca4227 $r$ index produces a corresponding uncertainty of up to \pm 0.05 dex in the derived Ca abundance.

(vi) While the $[\mathrm{Ca} / \mathrm{Fe}]$ abundances in early-type galaxies, as determined from the $\mathrm{Ca} 4227_{r}$ index, are now consistent with those of $[\mathrm{Mg} / \mathrm{Fe}]$, there remains an unresolved inconsistency between $[\mathrm{Ca} / \mathrm{Fe}]$ derived from the Ca I 4227 feature and that determined from the IR Ca triplet (Thomas et al. 2003 and references therein). That problem is reinforced by the recent result of Cenarro et al. (2004) that the Ca abundances inferred from the Ca4227 feature versus those inferred from the Ca II triplet exhibit different trends in cluster versus field galaxies. Clearly, there remain unresolved issues before definitive $\mathrm{Ca}$ abundances can be extracted from the integrated spectra of galalxies.

We thank Kristi Concannon and Nelson Caldwell for allowing us to utilize the CRC galaxy data. This study was partially funded by NSF grant AST-0406443 to the University of North Carolina. R.P.S. acknowledges financial support from HST Treasurey Program grant GO-09455.05-A to the University of Virginia.

\section{REFERENCES}

Alloin, D., Bica, E. 1989, A\&A, 217, 57.

Caldwell, N., Rose, J. A., \& Concannon, K. D. 2003, AJ, 125, 2891. (CRC)

Carreta, E., Cohen, J. G., Gratton, R. G., \& Behr, B. B. 2001, AJ, 122, 1469

Carretta, E., Gratton, R. G., Bragaglia, A., Bonifacio, P., \& Pasquini, L. 2004, A\&A, 416, 925.

Cenarro, A. J., Cardiel, N., Gorgas, J., Peletier, R. F., Vazdekis, A., \& Prada, F. 2001, MNRAS, 326,959 .

Cenarro, A. J., Sanchez-Blazquez, P., Cadiel, N., \& Gorgas, J. 2004, ApJ, 614, 101.

Cohen, J. G. 1979, ApJ, 22, 405

Davies, R. L., Sadler, E. M., \& Peletier, R. F. 1993, MNRAS, 262, 650.

Edvardsson, B., Andersen, J., Gustafsson, B., Lambert, D. L., Nissen, P E., \& Tomkin, J. 1993, A\&A, 275, 101. 
Faber, S. M. \& French, H. B. 1980, ApJ, 235, 405.

Fabricant, D., Cheimets, P., Caldwell, N., \& Geary, J. 1998, PASP, 110, 79-85

Girardi, I., Bressan, A., Bertelli, G., \& Choisi, C. 2000, A\&AS, 141, 371.

Henry, R. B. C., \& Worthey, G. 1999, PASP, 111, 919.

Idiart, T. P., Thevenin, F., \& De Freitas Pacheco, J. A. 1997, AJ, 113, 1066.

Jones, L. A., PhD Thesis, University of North Carolina.

Jorgensen, I. 1997, MNRAS, 288, 161.

Kuntschner, H. 2000, MNRAS, 315, 184.

McWilliam, A., \& Rich, R. M. 1994, ApJS, 91, 749

Matteucci, F. 1994, A\&A, 288, 57

Norris, J., \& Freeman, K. C. 1979, ApJ, 230, L179

Origlia, L., Valenti, E., \& Rich, R. M. 2005, MNRAS, 356, 1276

Peletier, R. F. 1989, Ph.D. Thesis, University of Groningen

Reddy, B. E., Tomkin, J., Lambert, D. L., \& Allende Prieto, C. 2003, MNRAS, 340, 304

Rose, J. A. 1994, AJ, 107, 206.

Salasnich, B., Girardi, L., Weiss, A., Choisi, C. 2000, A\&A, 361,1023.

Schiavon, R. P., Barbuy, B., Bruzual, A. G. 2000, ApJ, 532, 453

Schiavon, R. P. 2005, ApJS, submitted

Thomas, D., Greggio, L., \& Bender, R. 1999, MNRAS, 302, 537.

Thomas, D., Maraston, C., \& Bender, R. 2003, MNRAS, 343, 279

Trager, S. C., Worthey, G., Faber, S. M., Burstein, D. \& Gonzalez, J. J. 1998, ApJS, 116, 1

Tripicco, M. J., \& Bell, R. A. 1995, ApJ, 110, 3035

Valdes, F., Gupta, R., Rose, J. A., Singh, H. P., \& Bell, D. J. 2004, ApJS, 152, 251.

Vazdekis A., Peletier, R. F., Beckman, J. E., \& Casuso, E. 1997, ApJS, 111, 203

Vazdekis, A., Cenarro, A. J., Gorgas, J., Cardiel, N., \& Peletier, R. F. 2003, MNRAS, 340, 1317.

Wheeler, J. C., Sneden, C., \& Truran, J. W. Jr. 1989, ARA\&A, 27, 279. 
Worthey, G., Faber, S. M., \& Gonzalez, J. J. 1992, ApJ, 398, 69.

Worthey, G., Faber, S. M., Gonzalwz, J, \& Burstein, D. 1994, ApJS, 94, 687.

Worthey, G., \& Ottaviani, D. L. 1997, ApJS, 111, 377.

Worthey, G. 1998, PASP, 110, 888.

Zoccali, M., Barbuy, B., \& Hill, V., Ortolani, S., Renzini, A., Bica, E., Momany, Y., Pasquini, L., Minniti, D., \& Rich, R. M. 2004, A\&A, 423, 507

This preprint was prepared with the AAS $\mathrm{LAT}_{\mathrm{E}} \mathrm{X}$ macros v5.2. 
Table 1. Index Definitions

\begin{tabular}{lll}
\hline \hline \multicolumn{1}{c}{ Index } & \multicolumn{1}{c}{ Index Passband } & \multicolumn{1}{c}{ Pseudocontinua } \\
\hline $\mathrm{Ca} 4227_{r}$ & $4221.0-4230.8$ & $4241.0-4257.0$ \\
$\mathrm{Ca} 4227$ (Worthey et al. 1994) $^{\mathrm{a}}$ & $4223.5-4236.0$ & $4212.25-4221.0,4242.25-4252.25$ \\
$\mathrm{Mg} b^{\mathrm{a}}$ & $5160.125-5192.625$ & $5142.625-5161.375,5191.375-5206.375$ \\
$\mathrm{Fe} 4383^{\mathrm{a}}$ & $4370.375-4221.625$ & $4360.375-4371.625,4444.125-4456.625$ \\
\hline
\end{tabular}

${ }^{a}$ Index definitions are from Worthey et al. (1994).

Table 2. Ca4227 $r$ and Lick Ca4227 Index Measurements (Jones Library Stars)

\begin{tabular}{cll}
\hline \hline Name & Ca4227 & Lick Ca4227 \\
\hline BD+01_2341 & -0.0829 & 0.0446 \\
BD+09_3223 & 0.1229 & 0.1607 \\
BD+11_2998 & 0.2014 & 0.2702 \\
BD+18_2890 & 0.2638 & 0.3686 \\
BD+18_2976 & 0.1453 & 0.1655 \\
BD+25_1981 & -0.0143 & 0.1327 \\
\hline
\end{tabular}

Note. - A portion of Table 2 is shown here for guidance.

Table 3. Ca4227 $r$ and Lick Ca4227 Index Measurements (Galaxies and 47 Tuc)

\begin{tabular}{lllll}
\hline \hline Galaxy ID & $\mathrm{Ca} 4227_{r}$ & \multicolumn{1}{c}{ $\pm 1 \sigma$} & Lick Ca4227 & \multicolumn{1}{c}{ $\pm 1 \sigma$} \\
\hline 47Tuc & 0.6411 & 0.0099 & 0.5522 & 0.01058 \\
A00368p25 & 1.1209 & 0.117251 & 0.9840 & 0.0942476 \\
A10025 & 1.2044 & 0.0602541 & 1.0070 & 0.0484328 \\
A15572p48 & 0.9717 & 0.117251 & 0.9511 & 0.0942476 \\
A6044p16 & 1.1940 & 0.0618826 & 1.0457 & 0.0497418 \\
A23302p29 & 1.3129 & 0.156335 & 1.0713 & 0.125663 \\
\hline
\end{tabular}

Note. - A portion of Table 3 is shown here for guidance. 


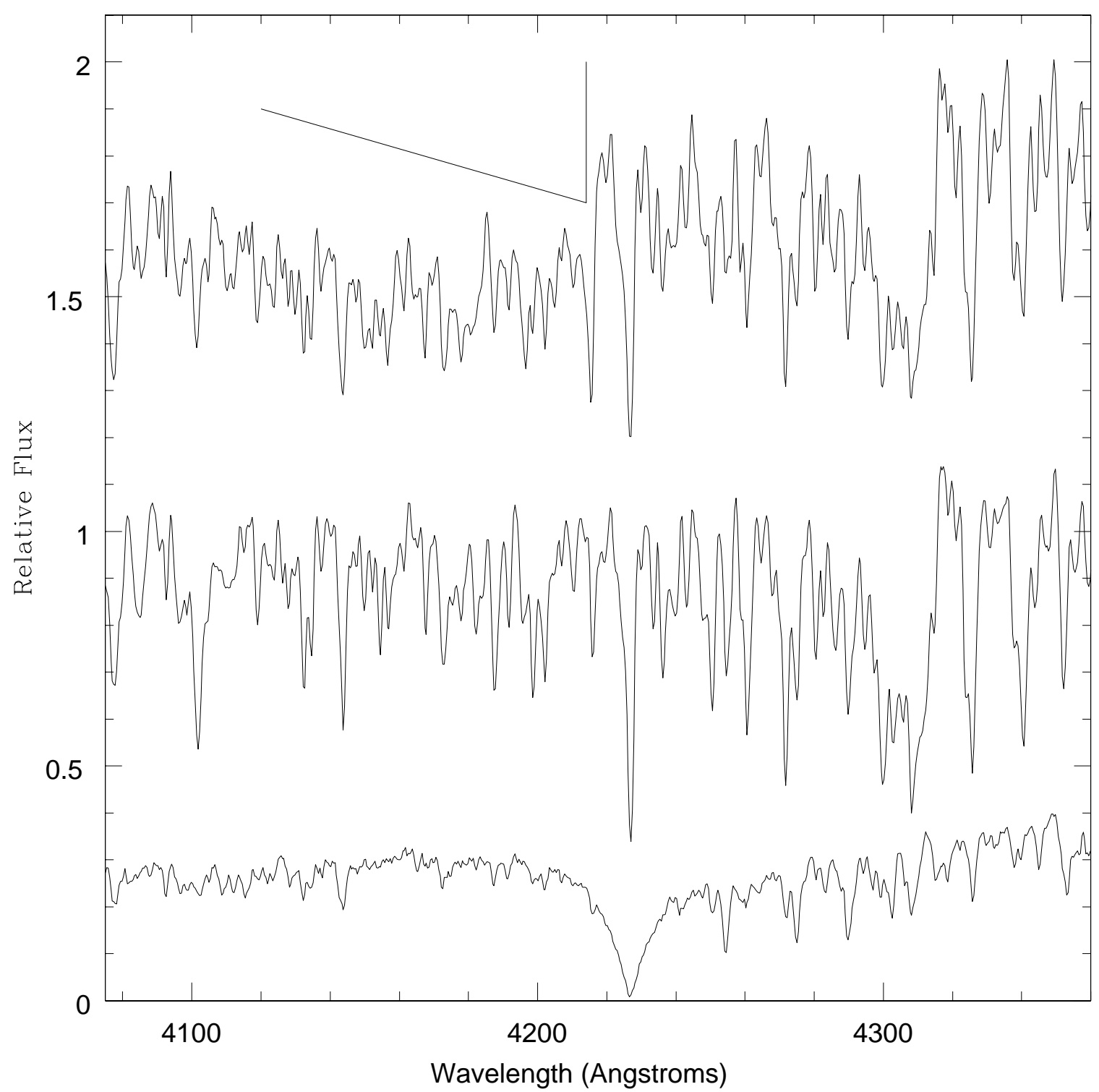

Fig. 1.- Representative spectra of three stars in the region of the Ca I 4227 absorption line illustrate the difficulty in defining an index that cleanly measures the feature. The bottom spectrum is of the M dwarf G227-46, the middle spectrum is of the G5 dwarf HD115617, and the top spectrum is of the K0III star HD115004. The wedge at the top illustrates the position of the CN4216 molecular band, with the bandhead at $4216 \AA$. All spectra have a resolution of $\sim 1.2 \AA$ FWHM. 


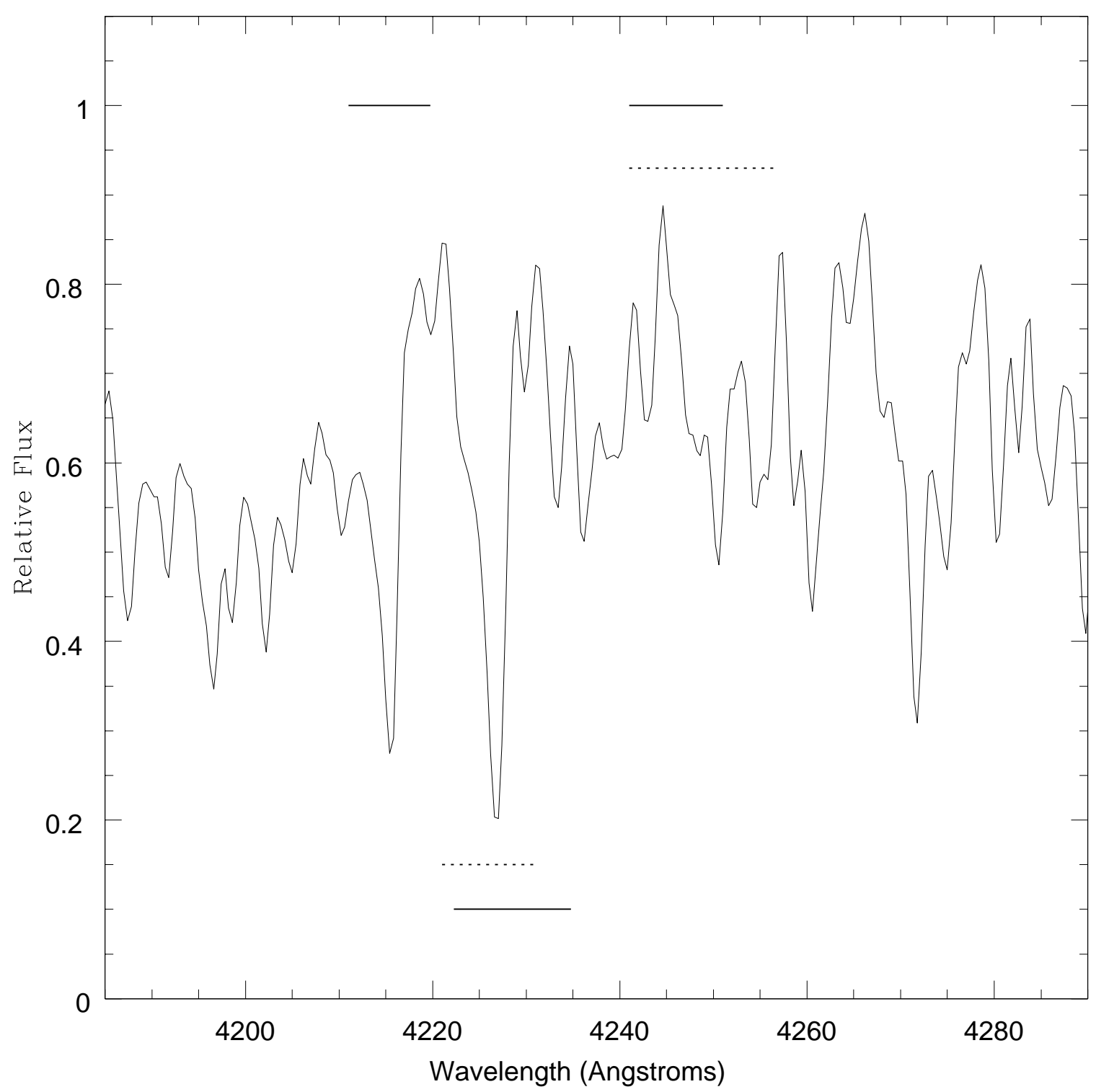

Fig. 2.- The spectrum of the same K0III star, HD115004, as in Fig. 1 is plotted in the vicinity of the Ca I 4227 absorption line, with dotted lines indicating the central bandpass and the red continuum bandpass used in our Ca4227 $7_{r}$ index. For comparison, the solid lines denote the central bandpass and blue and red continuum bandpasses of the Lick Ca4227 index. 


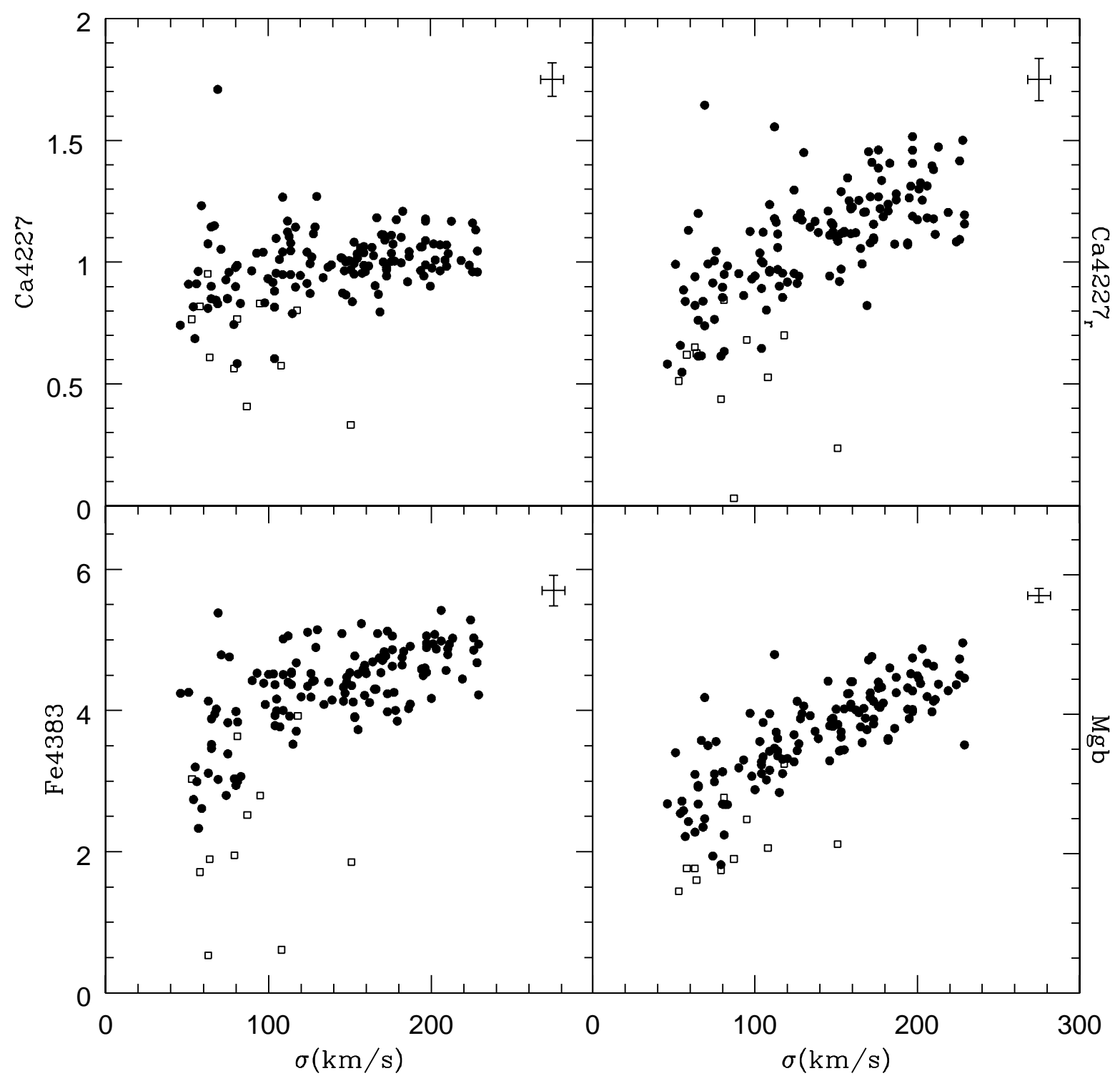

Fig. 3.- Empirical trends of $\mathrm{Ca}, \mathrm{Mg}$, and $\mathrm{Fe}$ versus galaxy velocity dispersion are presented for the CRC integrated spectra of early-type galaxies. The open squares denote young galaxies (EWH $\beta$ $\gtrsim$ 3.0). In the top left panel are plotted data for the Lick Ca4227 index, while that for our newly defined Ca4227 $r$ index is shown in the top right panel. For reference, the trends for the $\mathrm{Mg} b$ and Fe4383 indices are plotted in the lower left and right panels respectively. Error bars represent the mean r.m.s. errors among repeated galaxy observations. 

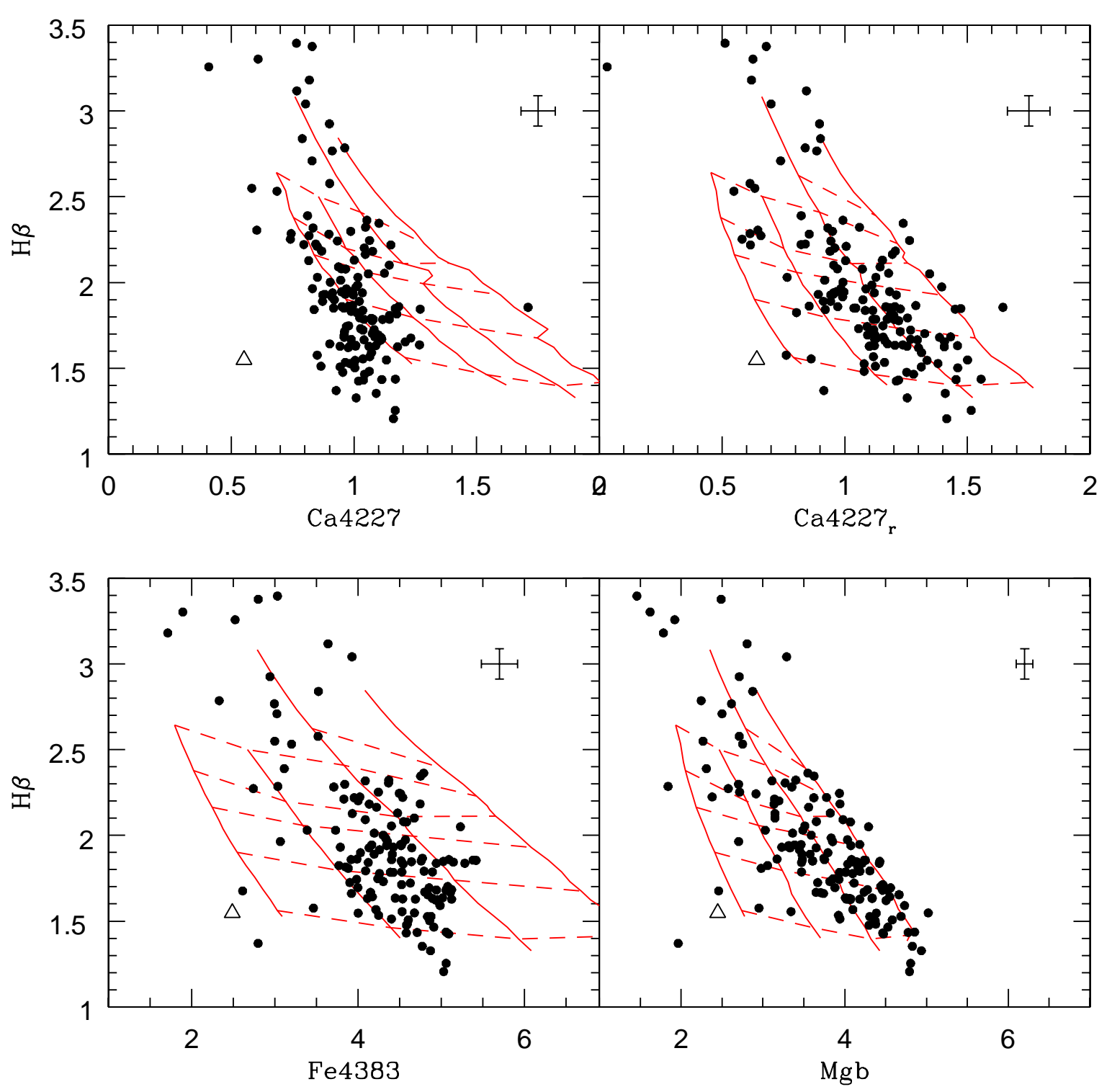

Fig. 4.- Lick Ca4227, Ca4227, $\mathrm{Mg} b$, and Fe4383 versus $\mathrm{H} \beta$ for the same CRC galaxy data as in Fig 3. Also plotted is a data point for the globular cluster 47 Tuc (open triangle). Overplotted on the data points are the model grid lines of constant age and $[\mathrm{Fe} / \mathrm{H}]$ from Schiavon (2005). The specific metallicities (solid lines) are, from left to right, $[\mathrm{Fe} / \mathrm{H}]=-0.8,-0.4,0.0$, and +0.3 . The ages (dashed lines) are, top to bottom, 2.0, 2.5, 3.5, 5.0, 7.9, and 14.1 Gyr. Error bars represent the mean r.m.s. errors among repeated galaxy observations. 


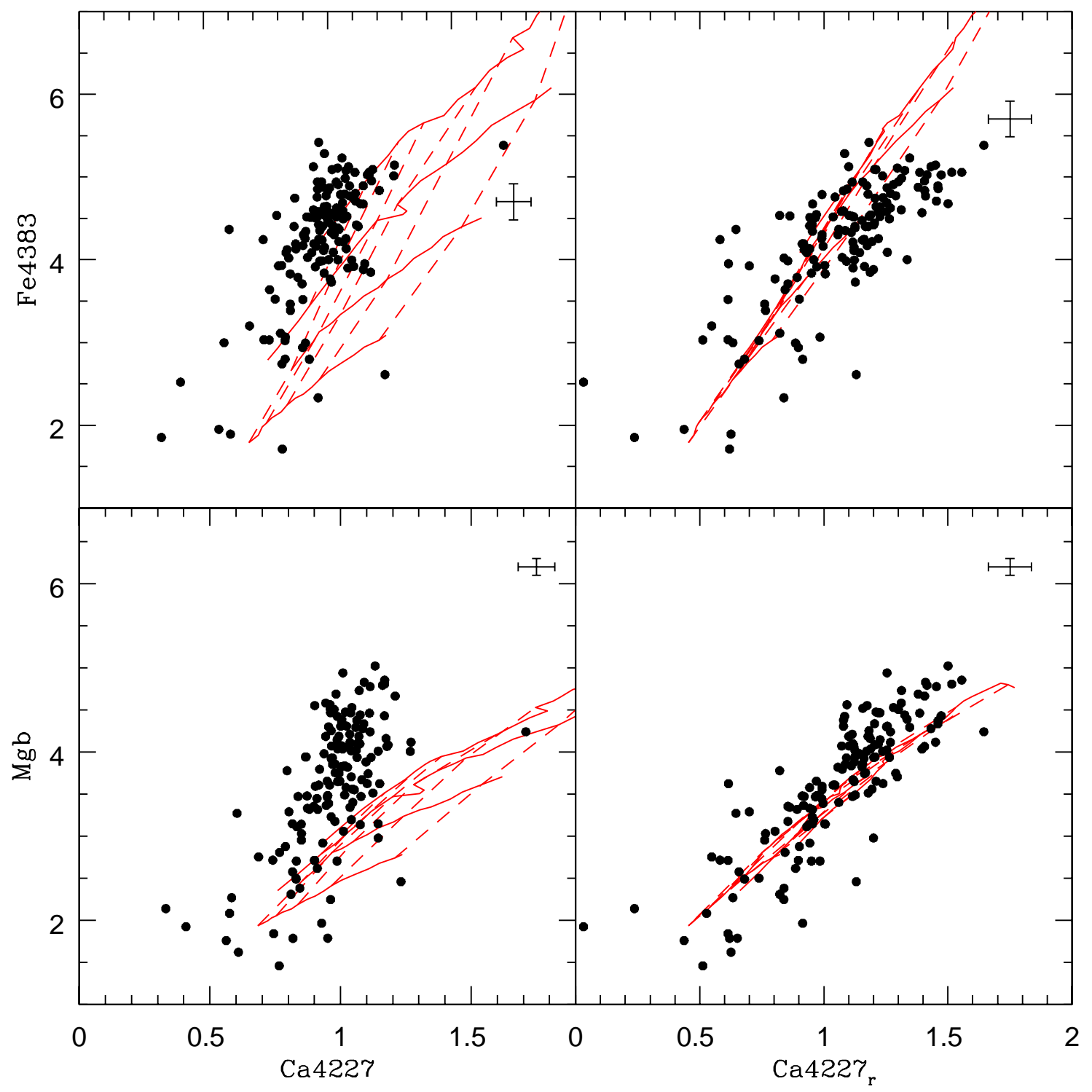

Fig. 5.- Fe4383 (top panels) and $\mathrm{Mg} b$ (bottom panels) galaxy data from CRC is plotted versus the Lick Ca4227 (left panels) and the new Ca4227 $r$ index (right panels). The model grid lines are the same as in Fig. 4. In the top left and bottom left panels, lines of constant age are increasing from left to right and metallicity increases from bottom up. Error bars represent the mean r.m.s. errors among repeated galaxy observations. 

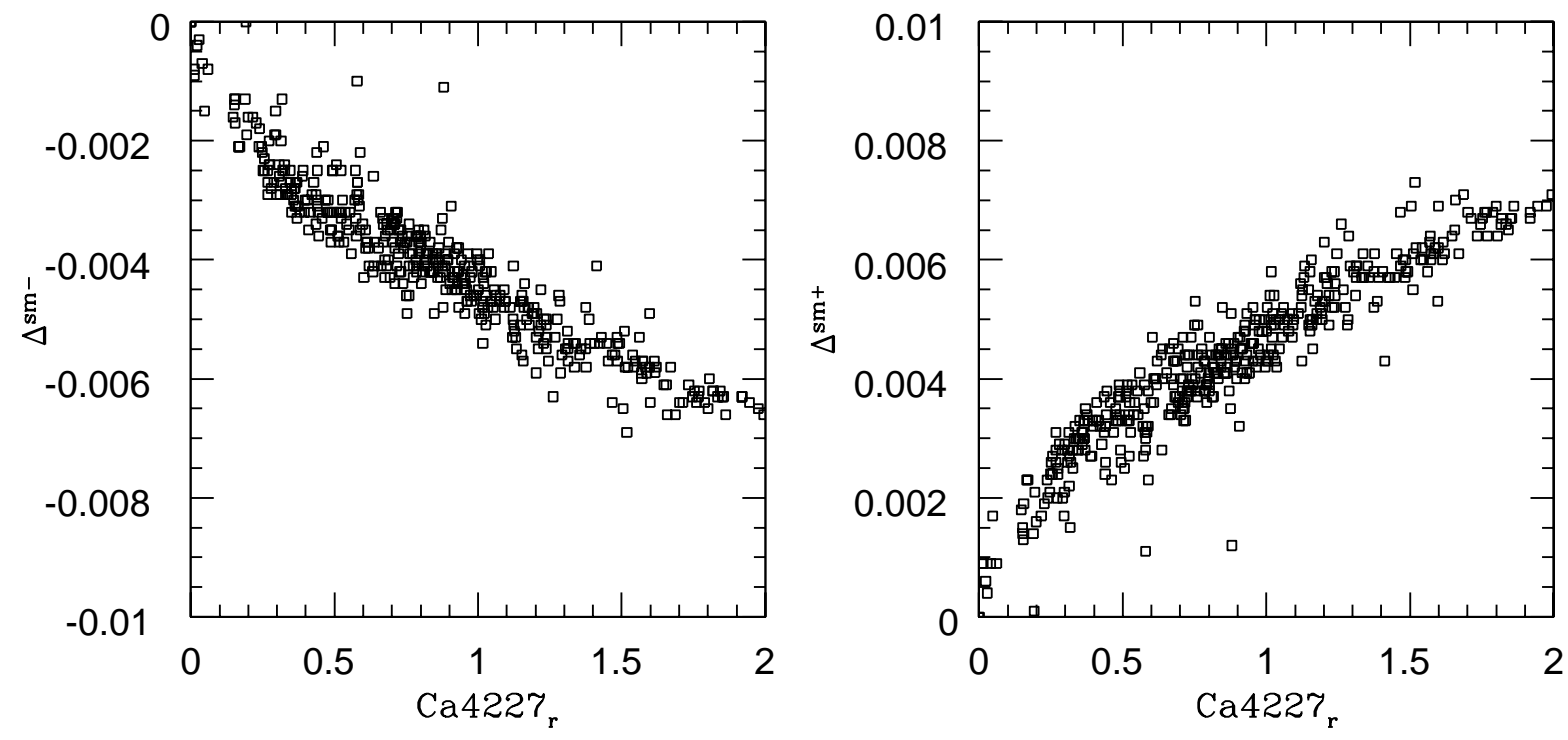

Fig. 6. - The effect of spectral resolution on the measured Ca4227 $r$ index is illustrated. In the left panel we plot the difference between the index measured at $3.1 \AA$ FWHM resolution with that at $2.95 \AA \mathrm{FWHM}$ (in the sense that $\Delta^{s m-}$ is the Ca4227 $r$ index at $3.1 \AA$ resolution minus Ca4227 $7_{r}$ at $2.95 \AA$ resolution). In the right panel, $\Delta^{s m+}$ is the Ca4227r index at $3.1 \AA$ resolution minus $\mathrm{Ca} 4227_{r}$ at $3.25 \AA$ resolution. 

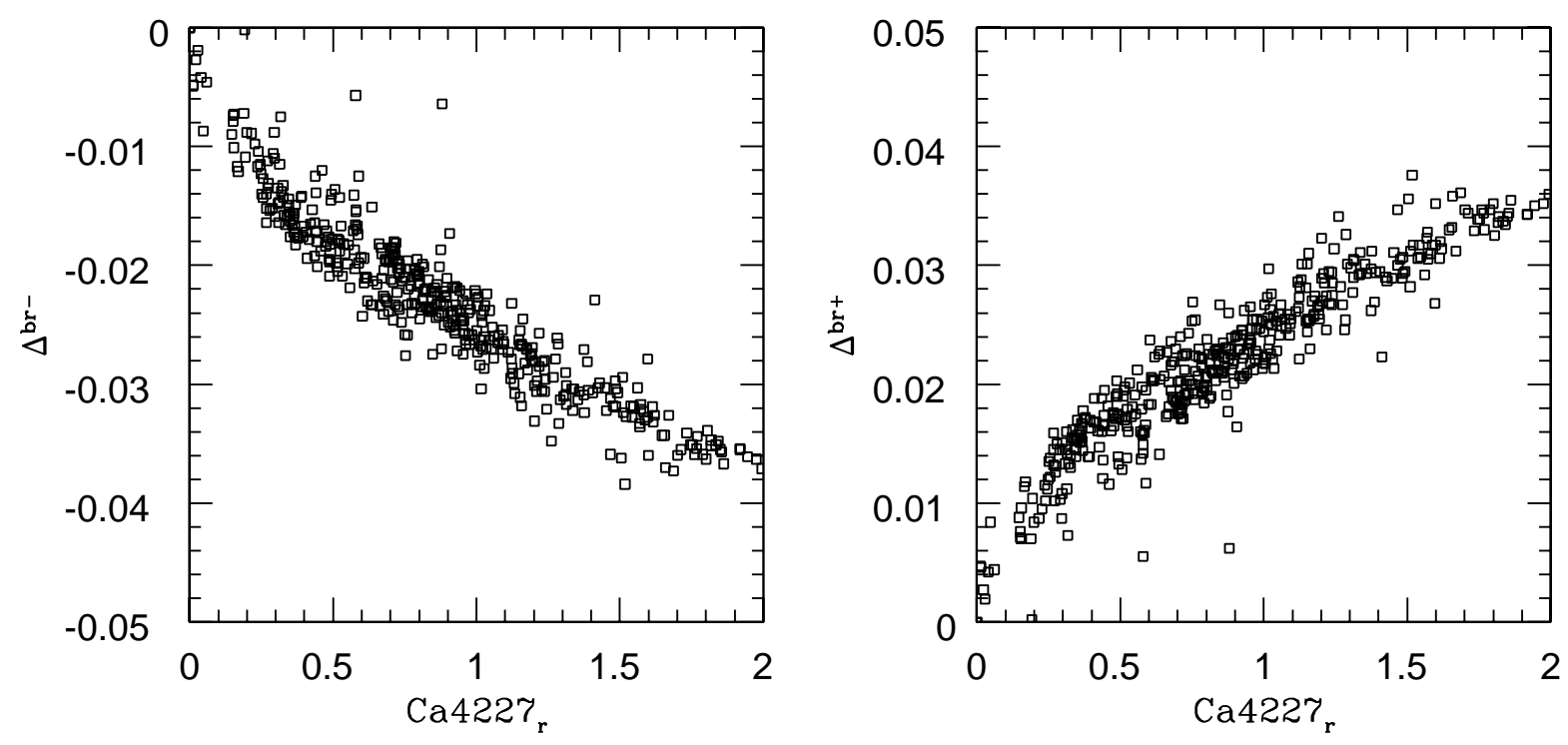

Fig. 7. - The effect of Doppler broadening on the measured Ca4227 $r$ index is illustrated. In the left panel we plot the difference between the index measured at a Doppler broadening, $\sigma$, of 230 $\mathrm{km} \mathrm{s}^{-1}$ with that broadened to $220 \mathrm{~km} \mathrm{~s}^{-1}$ (in the sense that $\Delta^{b r-}$ is the Ca4227 $r$ index at $\sigma$ of $230 \mathrm{~km} \mathrm{~s}^{-1}$ minus Ca4227 $r$ at $220 \mathrm{~km} \mathrm{~s}^{-1}$ broadening). In the right panel, $\Delta^{b r+}$ is the Ca4227 $r$ index at a $\sigma$ of $230 \mathrm{~km} \mathrm{~s}^{-1}$ minus that at $\sigma$ of $240 \mathrm{~km} \mathrm{~s}^{-1}$. 


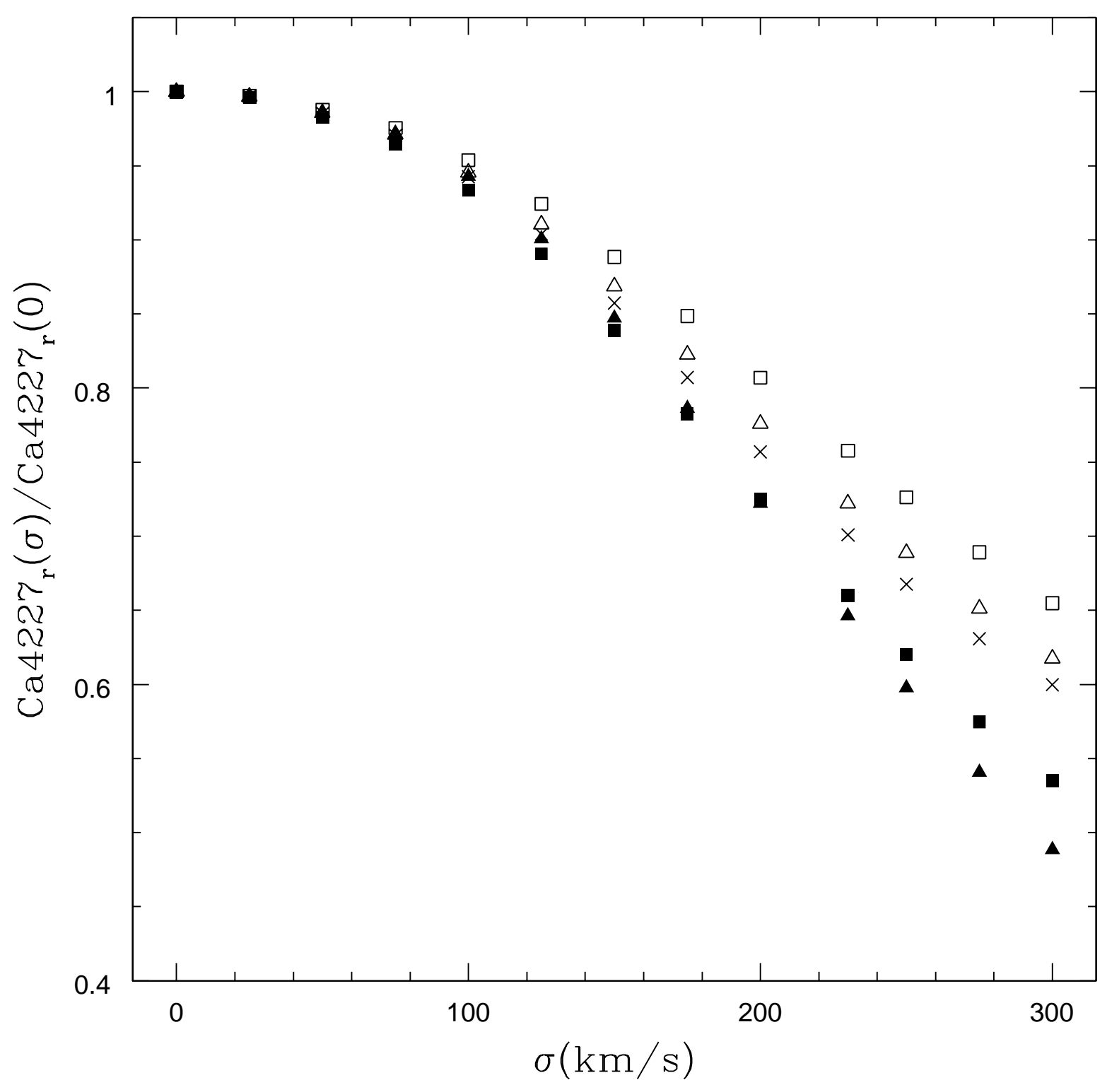

Fig. 8. - The dependence of the new Ca4227 $r$ index on galaxy velocity dispersion is illustrated. The $\mathrm{Ca} 4227_{r}$ index, normalized to Ca4227 $r$ at $\sigma=0 \mathrm{~km} \mathrm{~s}^{-1}$, is plotted against the velocity dispersion, $\sigma$. Results are plotted for 5 stellar spectra, covering a range in $\mathrm{Ca} 4227_{r}$ index. The stellar spectra are designated by open square, open triangle, cross, closed square, and closed triangle representing in increasing order a run in the Ca4227 $r$ index from 0.47 to 1.57 . 

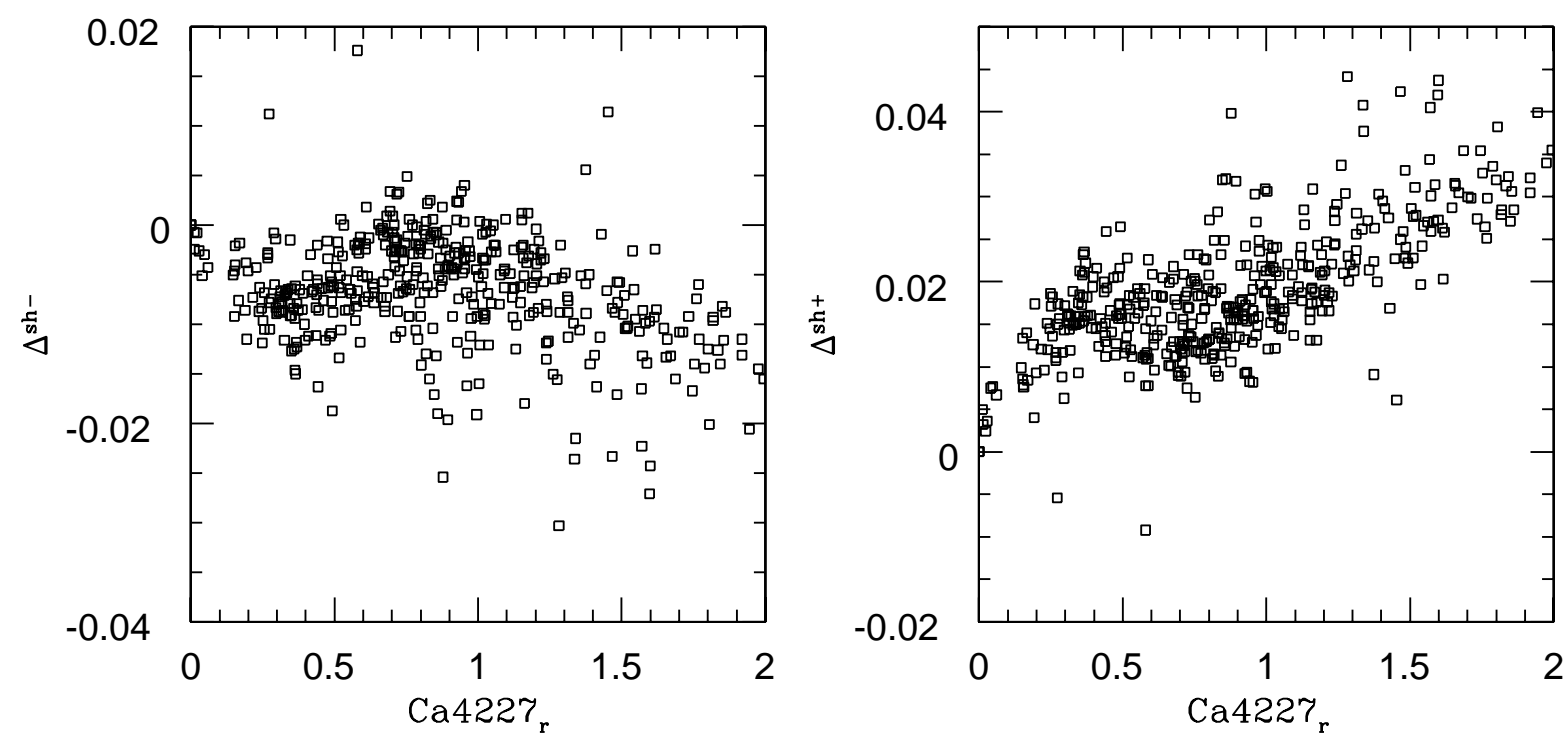

Fig. 9.- The effect of a wavelength zero point shift on the measured Ca4227 $r$ index is illustrated. In the left panel, the wavelength scale has been shifted by $-0.5 \AA$, and the difference in the measured

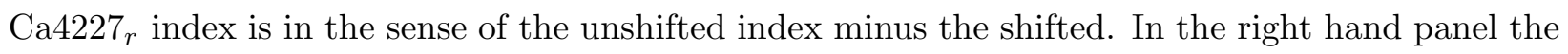
same procedure has been carried out, except that that a shift of $+0.5 \AA$ has been carried out. 